\title{
Cosmic Microwave Background bispectrum from primordial magnetic fields on large angular scales
}

\author{
T. R. Seshadri \\ Department of Physics and Astrophysics, University of Delhi, Delhi 110007, Indid \\ Kandaswamy Subramanian \\ IUCAA, Post Bag 4, Ganeshkhind, Pune 411 007, India
}

(Dated: August 27, 2018)

\begin{abstract}
Primordial magnetic fields lead to non-Gaussian signals in the Cosmic Microwave Background (CMB) even at the lowest order, as magnetic stresses, and the temperature anisotropy they induce, depend quadratically on the magnetic field. In contrast, CMB non-Gaussianity due to inflationary scalar perturbations arise only as a higher order effect. We propose here a novel probe of stochastic primordial magnetic fields that exploits the characteristic CMB non-Gaussianity that they induce. In particular, we compute the CMB bispectrum $\left(b_{l_{1}} l_{2} l_{3}\right)$ induced by stochastic primordial fields on large angular scales. We find a typical value of $l_{1}\left(l_{1}+1\right) l_{3}\left(l_{3}+\right.$

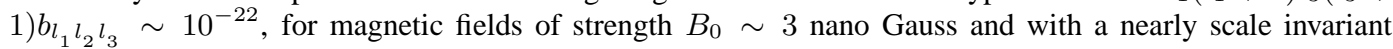
magnetic spectrum. Current observational limits on the bispectrum allow us to set upper limits on $B_{0} \sim 35$ nano Gauss, which can be improved by including other magnetically induced contributions to the bispectrum.
\end{abstract}

PACS numbers: 98.62.En, 98.70.Vc, 98.80.Cq, 98.80.Jk

Magnetic fields are ubiquitous in the universe but their origin and evolution is still not fully understood. A popular paradigm is that the observed magnetic fields result from the dynamo amplification of small seed fields. Astrophysical dynamos have been extensively studied [1] but are not without their difficulties. Another interesting alternative is that the observed large-scale magnetic fields have their origin in the early Universe, arising perhaps during inflation or some other phase transition [2]. If a primordial magnetic field with a present-day strength of a few nano Gauss is generated in the early universe, it would strongly affect the temperature and polarization anisotropies of the Cosmic Microwave Background Radiation. Such anisotropies arise both due to metric perturbations induced by magnetic stresses as well as fluid motions due to the Lorentz force [3, 4, 5]. We study here a new probe of primordial magnetic fields, namely, the possible non-Gaussian signals they induce in the CMB.

Previous work on non-Gaussian signals from a cosmological magnetic field has focused on a homogeneous magnetic field [6]. Such a field can result in correlations breaking spatial isotropy and also possibly result in north-south anisotropy on the CMB sky. We focus here on stochastic primordial fields which are statistically isotropic and homogeneous, and consider the non-Gaussian signals they induce.

Non-Gaussianity of the temperature anisotropy of the CMB has recently aroused considerable interest in the context of inflationary models. In such models, linearized quantum fluctuations of the inflaton on sub-Hubble scales lead eventually to classical long wavelength curvature perturbations. The Gaussian statistics of the initial quantum fluctuations lead to Gaussian statistics for the curvature perturbations and the induced CMB anisotropies. Non-Gaussianity can come in these models only through higher order effects. The magnetic stresses on the other hand, depend quadratically on the field. Hence, even for the field with Gaussian probability distribution, the magnetic stresses have necessarily a non-Gaussian character even at the lowest order. This will naturally lead to nonGaussianity in the CMB anisotropies induced by primordial fields. Thus bounds on CMB non-Gaussianity can strongly constrain primordial magnetic fields, or lead to their detection.

The CMB bispectrum or the 3-point function of the CMB anisotropies, if non-zero, provides an important and useful characterization of CMB non-Gaussianity. It contains more information, for example, than the single point probability distribution function (PDF). We compute here the simplest contribution to the CMB bispectrum, namely that due to the magnetically induced Sachs-Wolfe type effects.

The magnetic field is assumed to be a Gaussian random field. On galactic scales and above, any velocity induced by Lorentz forces is generally so small that it does not lead to appreciable distortion of the initial field [7]. So, the magnetic field simply redshifts away as $\mathbf{B}(\mathbf{x}, t)=\mathbf{b}_{0}(\mathbf{x}) / a^{2}$, where, $\mathbf{b}_{0}$ is the magnetic field at the present epoch (i.e. at $z=0$ or $a=1$ )

The magnetically induced Sachs-Wolfe type contribution to the temperature anisotropies given in [8, 9], which arises on large-angular scales, can be expressed as,

$$
\frac{\Delta T}{T}(\boldsymbol{n})=\mathcal{R} \Omega_{B}\left(\boldsymbol{x}_{0}-\boldsymbol{n} D^{*}\right) .
$$

Here $\Omega_{B}(\mathbf{x})=\mathbf{B}^{2}(\mathbf{x}, t) /\left(8 \pi \rho_{\gamma}(t)\right)=\mathbf{b}_{0}^{2}(\mathbf{x}) /\left(8 \pi \rho_{0}\right)$, where $\rho_{\gamma}(t)$ and $\rho_{0}$ are respectively the CMB energy densities at times $t$ and at the present epoch. (Note that the $\Delta T / T$ given above is obtained for on large-angular scales, just as the usual Sachs-Wolfe effect.) Ref. [8] gives an analytic estimate $\mathcal{R}=$ $R_{\gamma} / 20$ as the Sachs-Wolfe contribution, where $R_{\gamma} \sim 0.6$ is the fractional contribution of radiation energy density towards the total energy density of the relativistic component. Further, the unit vector $\mathbf{n}$ gives the direction of observation and $D^{*}$ is the (angular diameter) distance to the surface of last scatter. We have assumed an instantaneous recombination which is a good approximation for large angular scales. Note that 
there could also be additional integrated Sachs-Wolfe (ISW) contributions to $\mathcal{R}$ [8].

The temperature fluctuations can be expressed in terms of the spherical harmonics to give $\Delta T(\boldsymbol{n}) / T=$ $\sum_{l m} a_{l m} Y_{l m}(\boldsymbol{n})$, where

$$
a_{l m}=4 \pi \frac{1}{i^{l}} \int \frac{d^{3} k}{(2 \pi)^{3}} \mathcal{R} \hat{\Omega}_{B}(\boldsymbol{k}) j_{l}\left(k D^{*}\right) Y_{l m}^{*}(\hat{\boldsymbol{k}}) .
$$

Here $\hat{\Omega}_{B}(\boldsymbol{k})$ is the Fourier transform of $\Omega_{B}(\mathbf{x})$. Since $\Omega_{B}(\mathbf{x})$ is quadratic in $\mathbf{b}_{0}(\mathbf{x})$, we have $\hat{\Omega}_{B}(\boldsymbol{k})=\frac{1}{(2 \pi)^{3}} \int d^{3} s b_{i}(\boldsymbol{k}+$ $\boldsymbol{s}) b_{i}^{*}(\boldsymbol{s}) /\left(8 \pi \rho_{0}\right)$, where now $b_{i}(\boldsymbol{k})$ is the Fourier transform of $\mathbf{b}_{0}(\mathbf{x})$.

A measure of non-Gaussianity in the CMB temperature anisotropy is its 3-point correlation function and is called the bispectrum, $B_{l_{1} l_{2} l_{3}}^{m_{1} m_{2} m_{3}}$. In terms of the $a_{l m}$ 's it is given by,

$$
B_{l_{1} l_{2} l_{3}}^{m_{1} m_{2} m_{3}}=<a_{l_{1} m_{1}} a_{l_{2} m_{2}} a_{l_{3} m_{3}}>\text {. }
$$

From Eq.2] we can express $B_{l_{1} l_{2} l_{3}}^{m_{1} m_{2} m_{3}}$ as

$B_{l_{1} l_{2} l_{3}}^{m_{n_{2} m_{2} m_{3}}}=\mathcal{R}^{3} \int\left[\prod_{i=1}^{3}(-i)^{l_{i}} \frac{d^{3} k_{i}}{2 \pi^{2}} j_{l_{i}}\left(k_{i} D^{*}\right) Y_{l_{i} m_{i}}^{*}\left(\hat{\boldsymbol{k}}_{i}\right)\right] \zeta_{123}$

with $\zeta_{123}$ defined as,

$$
\zeta_{123}=<\hat{\Omega}_{B}\left(\boldsymbol{k}_{1}\right) \hat{\Omega}_{B}\left(\boldsymbol{k}_{2}\right) \hat{\Omega}_{B}\left(\boldsymbol{k}_{2}\right)>.
$$

The magnetic field itself is assumed to be non-helical and to have a Gaussian probability distribution. Hence it is completely specified by its energy power spectrum say $M(k)$. This spectrum is defined by the relation $<b_{i}(\mathbf{k}) b_{j}^{*}(\mathbf{q})>=$ $(2 \pi)^{3} \delta(\mathbf{k}-\mathbf{q}) P_{i j}(\mathbf{k}) M(k)$, where $P_{i j}(\mathbf{k})=\left(\delta_{i j}-k_{i} k_{j} / k^{2}\right)$ is the projection operator ensuring $\nabla \cdot \mathbf{b}_{\mathbf{0}}=0$. This gives $<\mathbf{b}_{0}^{2}>=2 \int(d k / k) \Delta_{b}^{2}(k)$, where $\Delta_{b}^{2}(k)=k^{3} M(k) /\left(2 \pi^{2}\right)$ is the power per logarithmic interval in $k$ space residing in the stochastic magnetic field.

As in [5], we assume a power-law magnetic spectra, $M(k)=A k^{n}$ that has a cutoff at $k=k_{c}$, where $k_{c}$ is the Alfvén-wave damping length-scale [7]. We fix $A$ by demanding that the variance of the magnetic field smoothed over a 'galactic' scale, $k_{G}=1 \mathrm{hMpc}^{-1}$, (using a sharp $k$-space filter) is $B_{0}$. This gives, (for $n>-3$ and for $k<k_{c}$ )

$$
\Delta_{b}^{2}(k)=\frac{k^{3} M(k)}{2 \pi^{2}}=\frac{B_{0}^{2}}{2}(n+3)\left(\frac{k}{k_{G}}\right)^{3+n} .
$$

The 3-point correlation function of $\hat{\Omega}_{B}(\boldsymbol{k})$ involves a 6 point correlation function of the fields. A long but tedious calculation gives $\zeta_{123}=\delta\left(\boldsymbol{k}_{1}+\boldsymbol{k}_{2}+\boldsymbol{k}_{3}\right) \psi_{123}$, where

$$
\psi_{123}=\frac{1}{\left(4 \pi \rho_{0}\right)^{3}} \int d^{3} s M\left(\left|\boldsymbol{k}_{1}+\boldsymbol{s}\right|\right) M(s) M\left(\left|\boldsymbol{s}-\boldsymbol{k}_{3}\right|\right) F .
$$

Here $F=\alpha^{2}+\beta^{2}+\gamma^{2}-\alpha \beta \gamma$ with $\alpha=\left(\widehat{\boldsymbol{s}} \cdot \widehat{\boldsymbol{s}+\boldsymbol{k}_{1}}\right)$, $\beta=\left(\widehat{\boldsymbol{s}} \cdot \widehat{\boldsymbol{s}-\boldsymbol{k}_{3}}\right)$ and $\gamma=\left(\widehat{\boldsymbol{k}_{1}+\boldsymbol{s}} \cdot \widehat{\boldsymbol{s}-\boldsymbol{k}_{3}}\right)$, where the hat on a vector denotes its unit vector. This result has also been obtained in Ref. [10].

We calculate the bispectrum in two limits: (i) the 'equilateral' case for which the three $l_{i}$ 's are equal, and, (ii) the 'local isosceles' case for which $l_{2}=l_{3} \gg l_{1}$. In the former case, the presence of $j_{l_{i}}\left(k_{i} D^{*}\right)$ in Eq. (4), predominantly picks out configurations in wave-number space, in the $\Omega_{B}$ bispectrum, with all the $k$ 's are almost equal. In the latter case, it picks out configurations in $k$ values with $k_{2} \sim k_{3} \gg k_{1}$.

The mode coupling integral can be usefully approximated in these two limits following methods discussed in earlier works [4, 5]. In case (i), when $k_{1}=k_{2}=k_{3}$, we split the $s$-integral into the sub-ranges $0<s<k_{1}$ and $s>k_{1}$. In each of these sub-ranges we approximate the mode-coupling integrand in Eq.7 by assuming that $s \ll k_{1}$ and $s \gg k_{1}$, respectively. Similarly, in the second case, when $k_{2}=k_{3} \gg k_{1}$, the $s$-integral is now split into sub-ranges, $0<s<k_{1}$, $k_{1}<s<k_{3}$ and $s>k_{3}$, and again in each of these subranges, we approximate the mode coupling integrand in Eq.7 by assuming that $s \ll k_{1}, k_{1} \ll s \ll k_{3}$ and $s \gg k_{3}$, respectively. We also restrict ourselves to spectral indices $-3<n<-3 / 2$. In fact blue spectra are strongly constrained by a number of observations, particularly the gravitational wave limits of Ref. [11]. For numerical estimates we will focus on nearly scale invariant spectra, that is $n \rightarrow-3$. For case (i) we then get

$\psi_{123}=\left(\frac{4}{3}\right)^{4} \frac{\pi^{7}}{k_{G}^{6}} \frac{(n+3)^{2}(7-n)}{2(|n+1|)}\left(\frac{k_{1}}{k_{G}}\right)^{2 n+3}\left(\frac{k_{3}}{k_{G}}\right)^{n} V_{A}^{6}$,

while for case (ii) we have

$$
\psi_{123}=\left(\frac{16}{3}\right)^{3} \frac{\pi^{7}}{k_{G}^{6}} \frac{(n+3)^{2}}{|2 n+3|}\left(\frac{k_{1}}{k_{G}}\right)^{2 n+3}\left(\frac{k_{3}}{k_{G}}\right)^{n} V_{A}^{6} .
$$

Here we have defined $V_{A}$, the Alfvén velocity in the radiation era as

$$
V_{A}=\frac{B_{0}}{\left(16 \pi \rho_{0} / 3\right)^{1 / 2}} \approx 3.8 \times 10^{-4} B_{-9}
$$

with $B_{-9} \equiv\left(B_{0} / 10^{-9}\right.$ Gauss $)$.

We now express the delta function in its integral form $\delta(\boldsymbol{k})=\left(1 /(2 \pi)^{3}\right) \int d^{3} x \exp (i \boldsymbol{k} \cdot \boldsymbol{x})$, use the spherical wave expansion of the exponential terms, substitute it into Eq. (4), and integrate over the angular parts of $\left(\boldsymbol{k}_{1}, \boldsymbol{k}_{2}, \boldsymbol{k}_{3}, \boldsymbol{x}\right)$. This algebra is very similar to what is done for calculating the primordial bispectrum [12]. After this algebra we can write the bispectrum $B_{l_{1} l_{2} l_{3}}^{m_{1} m_{2} m_{3}}$, in terms of a reduced bispectrum $b_{l_{1} l_{2} l_{3}}$ as

$$
B_{l_{1} l_{2} l_{3}}^{m_{1} m_{2} m_{3}}=\mathcal{G}_{m_{1} m_{2}}^{l_{1} l_{2} l_{3} m_{3}} b_{l_{1} l_{2} l_{3}}
$$


where

$$
\begin{aligned}
b_{l_{1} l_{2} l_{3}} & =\left(\frac{\mathcal{R}}{\pi^{2}}\right)^{3} \int x^{2} d x \\
& \times \prod_{i=1}^{3} \int k_{i}^{2} d k_{i} j_{l_{i}}\left(k_{i} x\right) j_{l_{i}}\left(k_{i} D^{*}\right) \psi_{123}
\end{aligned}
$$

and we have introduced the Gaunt integral

$$
\mathcal{G}_{m_{1} m_{2} m_{3}}^{l_{1} l_{2} l_{3}}=\int d \Omega Y_{l_{1} m_{1}} Y_{l_{2} m_{2}} Y_{l_{3} m_{3}} .
$$

For case (i), we substitute Eq. (8) into Eq. (12) for the reduced bispectrum. Similarly for case (ii) we substitute Eq. (9) into Eq. (12). The integrals over $k_{2}$ can be immediately done using $\int k_{2}^{2} d k_{2} j_{l_{2}}\left(k_{2} x\right) j_{l_{2}}\left(k_{2} D^{*}\right)=\left(\pi / 2 x^{2}\right) \delta\left(x-D^{*}\right)$, and the delta function makes the $x$-integral trivial. We are then left with integrals over $k_{1}$ and $k_{3}$ given by

$$
\begin{aligned}
b_{l_{1} l_{2} l_{3}} & =\frac{\pi}{2}\left(\frac{\mathcal{R}}{\pi^{2}}\right)^{3} V_{A}^{6}\left[\int \frac{d k_{3}}{k_{3}} j_{l_{3}}^{2}\left(k_{3} D^{*}\right)\left(\frac{k_{3}}{k_{G}}\right)^{n+3}\right] \\
& \times\left[\int \frac{d k_{1}}{k_{1}} j_{l_{1}}^{2}\left(k_{1} D^{*}\right)\left(\frac{k_{1}}{k_{G}}\right)^{2(n+3)}\right] C(n) .
\end{aligned}
$$

Here for case (i)

$$
C(n)=\left(\frac{4}{3}\right)^{4} \frac{\pi^{7}}{2} \frac{(n+3)^{2}(7-n)}{(|n+1|)}
$$

where as for case (ii)

$$
C(n)=\left(\frac{16}{3}\right)^{3} \pi^{7} \frac{(n+3)^{2}}{|2 n+3|} .
$$

Note that for any $n$ we can evaluate the integral in Eq. (14) analytically in terms of Gamma functions. For power law spectra, the form of the integrals is the same as the usual Sachs-Wolfe term. We focus on the nearly scale invariant case, $n \approx-3$. Such magnetic spectra are expected to arise in inflationary models for primordial magnetic field generation that we assume here [2]. Further, let us first consider the purely Sachs-Wolfe contribution as in [8], which gives $\mathcal{R}=R_{\gamma} / 20 \sim 0.03$. For the equilateral case we then have

$$
l_{1}\left(l_{1}+1\right) l_{3}\left(l_{3}+1\right) b_{l_{1} l_{2} l_{3}} \approx 2.3 \times 10^{-23}\left(\frac{n+3}{0.2}\right)^{2}\left(\frac{B_{-9}}{3}\right)^{6}
$$

while for the local-isosceles case we get

$l_{1}\left(l_{1}+1\right) l_{3}\left(l_{3}+1\right) b_{l_{1} l_{2} l_{3}} \approx 1.5 \times 10^{-22}\left(\frac{n+3}{0.2}\right)^{2}\left(\frac{B_{-9}}{3}\right)^{6}$.

Including an ISW contribution can lead to larger signals. We remark that for $n \sim-3$, the numerical values of $l_{1}\left(l_{1}+\right.$ 1) $l_{3}\left(l_{3}+1\right) b_{l_{1} l_{2} l_{3}}$ is higher for the local isosceles case by a factor $\sim 6.4$, compared to the the equilateral case. The relative strengths of the bispectrum for the equilateral and local case has its origin in the ratio of $\psi_{123}$ for these two cases. It can indeed be seen from equations 8 and 9 that $\psi_{123(\text { local })}=\psi_{123(\text { equil })}(96|n+1|) /(|2 n+3|(7-n))$ which for $n \sim-3$ comes out to be $\sim 6.4 \psi_{123(\text { equil) }}$.

These values for the reduced bispectrum, should be compared with a value $l_{1}\left(l_{1}+1\right) l_{3}\left(l_{3}+1\right) b_{l_{1} l_{2} l_{3}} \sim 4 \times 10^{-18} f_{N L}$ at large angular scales, which arises due to nonlinear terms in the gravitational potential, characterized by $f_{N L}$ (cf. [13]). Thus the magnetically induced non-Gaussian signal, due to the purely Sachs-Wolfe effect (with $\mathcal{R}$ as in [8]), is a factor of about a few times $10^{4}$ smaller than the standard signal predicted in inflationary models with $f_{N L} \sim 1$. Conversely if observations constrain $l_{1}\left(l_{1}+1\right) l_{3}\left(l_{3}+1\right) b_{l_{1} l_{2} l_{3}}<4 \times 10^{-16}$, assuming $f_{N L}<100$ (for example say from WMAP experiment [14, 15]), then we have a limit of $B_{0}<35$ nano Gauss on the strength of any primordial field with a nearly scale invariant spectrum. Stronger limits would be obtained if we were to use potentially larger contributions from the ISW effect.

We note that the $l_{i}$ dependence for the standard primordial contribution to the bispectrum on large angular scales (for scale invariant potential perturbations) is the same as that we get here, in both cases (i) and (ii), for magnetically induced Sachs-Wolfe effect (for scale-invariant magnetic spectra) (cf. [12, 13]). That is in all these cases $l_{1}\left(l_{1}+1\right) l_{3}\left(l_{3}+1\right) b_{l_{1}, l_{2} l_{3}}$ is independent of $l$ 's (see Eq. (17) and (18) and Ref. [13]). Thus the bounds on $f_{N L}$ got from searching the WMAP data for the standard 'local' non-Gaussianity are indeed useful to set constraints on $B_{0}$. The WMAP limits use the much larger range of $l$ values than the range for which the SW contribution is important. However the limit on $B_{0}$ depends only very weakly $\left(B_{0} \propto f_{N L}^{1 / 6}\right)$ on the exact observational limit on $f_{N L}$. Thus our limits on $B_{0}$ are expected to be reasonably robust.

A systematic, statistical study of limits on $B_{0}$ from the CMB power spectrum alone, taking account of all the available data and comparing with the sum of scalar, vector and tensor modes of the magnetically induced signals, although challenging, would be of great interest. Present works deal with only a subset of the data or the signals: In typical models a field $B_{0} \sim 3 \mathrm{nG}$ for scale invariant spectra, are not excluded by any current CMB data (cf. [3, 4, 5, 9] and the reviews [16]), while $B_{0} \sim 10 \mathrm{nG}$ would seem to be excluded. In this paper we have attempted to probe stochastic primordial magnetic fields, arising from SW effect. The present constraint on $B_{0}$ derived above from the non-Gaussianity limits arises from the magnetically induced SW contribution to the bispectrum. Although the SW contribution above is weaker than that expected from inflationary contribution, it is of interest as this is a novel probe of stochastic primordial magnetic fields. This is of value for setting the stage for calculating the full contribution by including vector as well tensor modes. Vector modes are expected to contribute on small angular scales (large $l$ ). Tensor modes could contribute over the same range of $l$-values considered here. Adding these contributions is expected to lead to stronger limits on $B_{0}$ from limits on CMB 
non-Gaussianity. It is worth noting that if only the magnetically induced SW contribution is taken into account even the limit on $B_{0}$ from the CMB power spectrum would be much weaker. We have focused on SW contribution of scalar modes here so as to calculate, as a first cut, the simplest contribution to non-Gaussian signals induced by stochastic primordial magnetic fields.

There is a distinct advantage of using bispectrum (as a probe for stochastic primordial magnetic fields) over the power spectrum. The magnetically induced signal being fundamentally non Gaussian, could be more easily distinguishable in the bispectrum. This is due to the fact that bispectrum arising due to magnetic contribution can in principle dominate that arising from, models of inflation with a small enough $f_{N L}$. On the other hand, the problem with the power spectrum arising due to magnetic contribution is that it is generally subdominant to those arising from inflation generated curvature perturbations, for nano Gauss fields and scale invariant spectra. These will hence require careful analysis to isolate the magnetic contribution. The probe using bispectrum developed in this paper is free of such limitations.

In conclusion we have studied a new probe of primordial magnetic fields, by computing the CMB bispectrum they induce on large angular scales. The magnetically induced signals on the CMB anisotropies have a necessarily a nonGaussian character, as the temperature fluctuations they induce depend quadratically on the field strength. The CMB bispectrum is an important and useful probe of this non-Gaussian nature. Earlier work has emphasized the role of the CMB angular power spectrum in constraining and detecting primordial fields. As we have argued here, the bispectrum will provide a new and independent handle for constraining such fields. Further numerical work, which also considers all the scalar, vector and tensor modes, will help to strengthen the constraints derived here.

Note added: After we had submitted this paper a preprint [17] appeared which confirms our main conclusions.

Acknowledgement: The authors thank the anonymous referees for their many useful suggestions that have helped in improving this paper.

* Electronic address: trs@ physics.du.ac.in

$\dagger$ Electronic address: kandu@iucaa.ernet.in

[1] H. K. Moffatt, Magnetic Field Generation in Electrically Conducting Fluids, Cambridge University Press, Cambridge (1978); F. Krause, K.-H. Rädler, Mean-field magnetohydrodynamics and dynamo theory, Pergamon Press, Oxford
(1980); A. A. Ruzmaikin, A. Shukurov and D. Sokoloff, Magnetic Fields of Galaxies, Kluwer, Dordrecht (1988); A. Brandenburg and K. Subramanian, Phys. Rep. 417, 1 (2005).

[2] M. Turner, L. M. Widrow, Phys. Rev. D. 37, 2743-2754 (1988); B. Ratra, ApJ, 391, L1 (1992); L. M. Widrow, Rev. Mod. Phys., 74, 775-823 (2002); M. Giovannini, "String theory and fundamental interactions", eds. M. Gasperini and J. Maharana, Lecture Notes in Physics, Springer, Berlin/Heidelberg (2007) (arXiv:astro-ph/0612378)

[3] J. D. Barrow, P. G. Ferreira and J. Silk, Phys. Rev. Lett., 78, 3610-3613 (1997); K. Subramanian and J. D. Barrow, Phys. Rev. Lett., 81, 3575-3578 (1998); R. Durrer, P. G. Ferreira, T. Kahniashvili, Phys. Rev. D., 61, 043001 (2000); A. Lewis, Phys. Rev. D., 70, 043011 (2004); T. Kahniashvili and B. Ratra, Phys. Rev. D, 71, 103006 (2005); M. Giovannini, Phys. Rev. D., 74, 063002 (2006); M. Giovannini and K. E. Kunze, Phys. Rev. D., 77, 063003 (2008); D. G. Yamazaki, K. Ichiki, T. Kajino, and G. J. Mathews, Phys. Rev. D., 77, 043005 (2008); F. Finelli, F. Paci and D. Paoletti, Phys. Rev. D., 78, 023510 (2008); M. Giovannini, arXiv:0902.4353 (2009).

[4] T. R. Seshadri and K. Subramanian, Phys. Rev. Lett., 87, 101301-1-101301-4 (2001); A. Mack, T. Kahniashvili and A. Kosowsky, Phys. Rev. D., 65, 123004 (2002)

[5] K. Subramanian, T. R. Seshadri and J. D. Barrow, Mon. Not. Roy. Astr. Soc., 344, L31-L35 (2003)

[6] G. Chen, P. Mukherjee, T. Kahniashvili, B. Ratra and Yun Wang, Astrophys.J., 611, 655 (2004); P. D. Naselsky, L-Y. Chiang, P. Olesen and O. V. Verkhodanov, Astrophys. J., 615, 45 (2004); A. Bernui and W.S. Hipolito-Ricaldi, Mon. Not. R. Astron. Soc., 389, 1453 (2008); T. Kahniashvili, G. Lavrelashvili and B. Ratra, Phys. Rev. D., 78, 063012 (2008)

[7] K. Jedamzik, V. Katalinic, and A. Olinto, Phys. Rev. D57, 3264 (1998). K. Subramanian and J. D. Barrow, Phys.Rev. D58 083502 (1998).

[8] M. Giovannini, PMC Physics A, 1:5 (2007); (doi:10.1186/1754-0410-1-5)

[9] D. Paoletti, F. Finelli and F. Paci, arXiv:0811.0230 (2008)

[10] I. Brown, and R. Crittenden, Phys. Rev. D, 72, 063002 (2005)

[11] C. Caprini, R. Durrer, Phys. Rev. D, 65, 3517 (2002)

[12] J. R. Fergusson and E. P. S. Shellard, Phys. Rev. D, 76, 083523 (2007)

[13] A. Riotto, The quest for Non-Gaussianity, Lect. Notes Phys., 738, 305 (2008)

[14] E. Komatsu et al., Astrophys. J. Suppl. 180, 330 (2009)

[15] The WMAP 5-year data gives limits on primordial nonGaussianity $-9<f_{N L}^{\text {local }}<111$ and $-151<f_{N L}^{\text {equil }}<253$ at the $95 \%$ confidence level for the local and equilateral models, respectively. Of course these limits use the much larger range of $l$ values than the range for which the SW contribution is important.

[16] K. Subramanian, Astron. Nach., 327, 333 (2005); R. Durrer, New Astron. Rev., 51, 275 (2007).

[17] C. Caprini, F. Finelli, D. Paoletti and A. Riotto, arXiv:0903.1420 (2009) 